\title{
Far-infrared optical absorption and reflectivity of a superconducting $\mathrm{NbN}$ film
}

\author{
K. E. Kornelsen, ${ }^{*}$ M. Dressel, and J. E. Eldridge \\ Department of Physics, University of British Columbia, Vancouver, British Columbia, Canada V6T 1Z1 \\ M. J. Brett and K. L. Westra \\ Department of Electrical Engineering, University of Alberta, Edmonton, Alberta, Canada T6G $2 G 7$
}

(Received 11 June 1991)

\begin{abstract}
We report measurements of bolometric absorption and absolute reflectivity of a thin film of $\mathrm{NbN}$ on a silicon substrate, above and below the superconducting transition temperature of $13.3 \mathrm{~K}$. The opening of the gap at an energy of $3.9 k_{B} T_{c}$ is seen clearly in both types of measurement. The data are compared with the predictions of the Mattis-Bardeen theory. The change in the reflectivity and the absorption at energies above $2 \Delta$ is larger than that calculated from the theory. By an analysis of the reflectivity data, it is shown that the theoretical values of the complex superconducting conductivity are too high.
\end{abstract}

\section{INTRODUCTION}

Thin films of superconducting $\mathrm{NbN}$ are of great current interest because of their very low surface resistance, which is comparable to that of $\mathrm{Nb}$, and relatively high values of $T_{c}(13-18 \mathrm{~K})$. This is important for microwave device applications. Films made from the recently discovered high- $T_{c}$ ceramic materials have so far exhibited higher surface resistances. A recent study by Oates et al. ${ }^{1}$ reported measurements of the temperature and frequency dependencies of the surface impedance of $\mathrm{NbN}$ thin films in the microwave region using a stripline resonator and cavity technique. They satisfactorily fitted the temperature dependence of the $35 \mathrm{GHz}$ surface resistance by use of the weak-coupling dirty-limit MattisBardeen theory, ${ }^{2}$ but they also noted that their sensitivity was insufficient to differentiate between weak and strong coupling. In the far-infrared region, which contains the energy gap, measurements of transmission through thin $\mathrm{NbN}$ films have been reported by Karecki, Pena, and Perkowitz. ${ }^{3}$ They found it impossible, however, to satisfactorily fit the data with a dirty-limit assumption in which the scattering time $\tau=0$ and the normal conductivity has no frequency dependence. Instead they used their fit to obtain a value of $\tau$. One of the important potential applications of $\mathrm{NbN}$ is in tunnel junctions, and measurements ${ }^{4,5}$ using these devices have also shown the energygap and phonon density of states.

We have recently ${ }^{6}$ used a bolometric method in which the absorption $A$ is measured directly in an attempt to measure the energy gap in the $10 \mathrm{~K}$ organic superconductor, (BEDT-TTF $)_{2} \mathrm{Cu}(\mathrm{NCS})_{2}$, where BEDT-TTF is bis(ethylenedithio) tetrathiafulvalene. These measurements showed no sign of a superconducting gap, so it was important to test our methods with a better understood conductor. Using the $\mathrm{NbN}$ samples, we have successfully observed the energy gap as a function of temperature in thin-film $\mathrm{NbN}$ with high resolution and good signal to noise. We then measured the power reflectivity and again saw the gap clearly. We found a large discrepancy with the predictions of the Mattis-Bardeen theory, ${ }^{2}$ as did Karecki, Pena, and Perkowitz ${ }^{3}$ but do not feel that a suitable scattering time resolves the issue. By fitting our reflectivity data, which contained interference fringes produced by internal reflections within the substrate, we extracted absolute values of the complex conductivity, which are lower than the theoretical ones.

\section{EXPERIMENT}

The samples are in the form of a 250-nm-thick sputtered thin film on a silicon or quartz substrate using a deposition process described by Vaneldik. ${ }^{7}$ The silicon substrate is $0.39 \mathrm{~mm}$ thick and was cut to a size of $5 \mathrm{~mm} \times 5 \mathrm{~mm}$ for our experiments. Visually, the film is very shiny and smooth. Measurements of dc conductivity gave $T_{c}$ equal to $13.3 \mathrm{~K}$.

For the bolometric measurements, one piece of the sample was mounted at the bottom of a highly reflecting copper light cone. The maximum angle of incidence was $20^{\circ}$. The sample was large enough to completely cover the light-cone exit so that no light could leak around the edges and be absorbed indirectly. Support of the sample was provided by four strands of 0.025 -mm-diam gold wire, each wire about $1.5 \mathrm{~mm}$ long, which were attached to the back of the sample using a small amount of epoxy. This gives the bolometer a thermal response time constant of about $30 \mathrm{~ms}$ at $10.8 \mathrm{~K}$. Also glued to the back of the silicon substrate was a small doped silicon thermometer, which was used to both obtain a bolometric absorption signal and to determine the sample temperature. The resistance of this thermometer is exponentially decreasing with increasing temperature, and bolometric sensitivity is limited to the temperature range below about $15 \mathrm{~K}$.

A standard ${ }^{4} \mathrm{He}$ cryostat was used for the bolometric measurements. A small resistor on the side of the sample mount was used as a heater for varying the sample temperature. In the first cooling of the sample, no cold filters were positioned in front of the sample and the minimum 
temperature was only $10.8 \mathrm{~K}$ due to the roomtemperature radiation. Absorption spectra were measured at this minimum temperature and above $T_{c}$ at 14.1 $\mathrm{K}$. On the second cooling, 77 and $4.2 \mathrm{~K}$ diamond scatter filters were installed, and the minimum sample temperature reached was $5 \mathrm{~K}$. The cold filters reduce the light intensity reaching the detector, and the upper temperature limit for reasonable sensitivity was then $10.8 \mathrm{~K}$. These cold filters restricted the far-infrared range to below 110 $\mathrm{cm}^{-1}$.

For the reflectivity measurements, two different $\mathrm{NbN}$ samples were mounted side by side next to an aluminum reference mirror on a single copper block. These three surfaces could be exchanged in the reflectivity module by translation of the cryostat cold stage. The angle of incidence was $15^{\circ}$. One of the superconducting $\mathrm{NbN}$ samples was cut from the same piece of tunneling junction material on silicon used for the absorption measurements, while the other was a newly deposited film on a quartz substrate. The cryostat was of the continuous-flow type. Details of the reflectivity apparatus have been published elsewhere. ${ }^{8}$

The spectrometer used for the bolometric measurements was a far-infrared Beckmann-RIIC FS720 stepand integrate Fourier-transform machine, while the reflectivity data were obtained using a Bruker IFS 113V rapid-scan infrared Fourier spectrometer.

\section{RESULTS}

Figure 1 shows the absorption $A$ vs wave number, as a function of temperature below $T_{c}$, ratioed with that measured at $14 \mathrm{~K}$ above $T_{c}$. The 14-K spectrum (not shown), when ratioed with a background obtained using a Golay

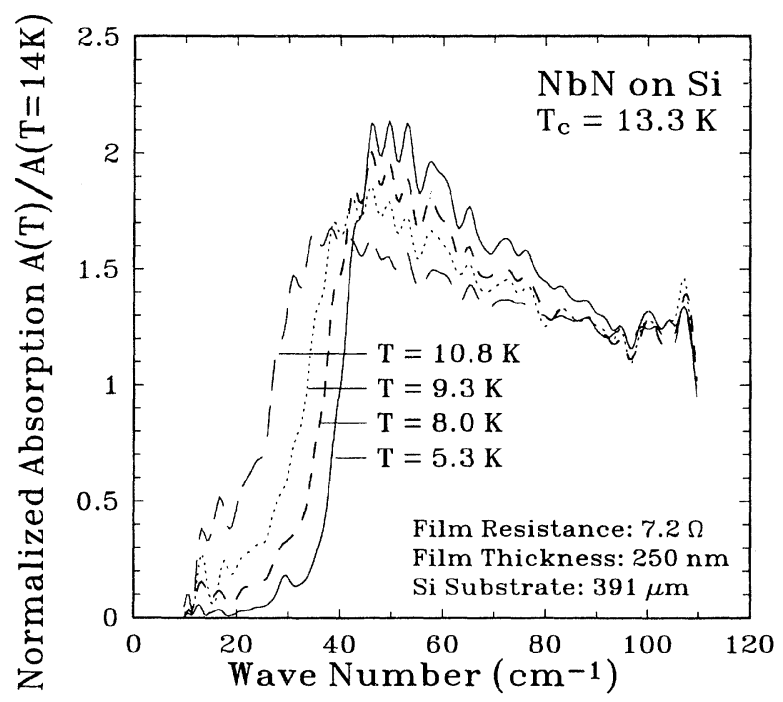

FIG. 1. The bolometric absorption spectra of a superconducting $\mathrm{NbN}$ film at four tempertures below $T_{c}$, ratioed with a 14-K spectrum above $T_{c}$. The curves have been scaled individually to agree at $110 \mathrm{~cm}^{-1}$ (for the purpose of comparison) and overall to give a 5.3-K spectrum, which equals 1.0 at large wave numbers. The resolution is $2 \mathrm{~cm}^{-1}$.

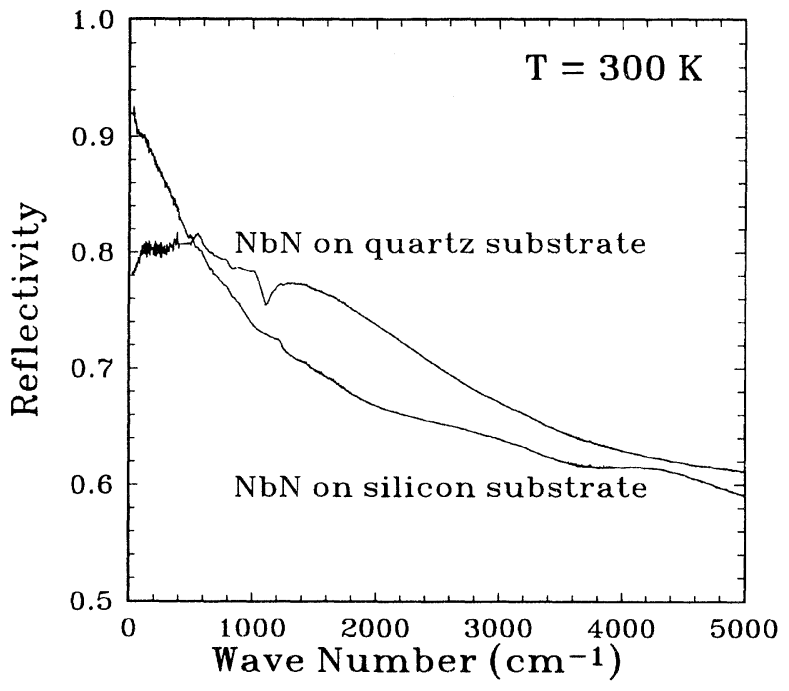

FIG. 2. The room-temperature reflectivity of two NbN thinfilm samples on different substrates. The quartz sample did not show an optical gap when cooled to $5 \mathrm{~K}$.

pneumatic detector, which has a flat wave-number response, is itself a flat constant response over this wavenumber range and shows no sign of the gap. The superconducting gap in Fig. 1 is seen clearly and has a strong temperature dependence. The $2-\mathrm{cm}^{-1}$ resolution shows the channelled spectra due to the interference in the silicon substrate. These data are not calibrated absolutely, however, since the sensitivity of the silicon bolometer is both temperaure-dependent and unknown (see figure caption).

An absolute optical response has been obtained in our measurements of the reflectivity, $R$. Figure 2 shows the

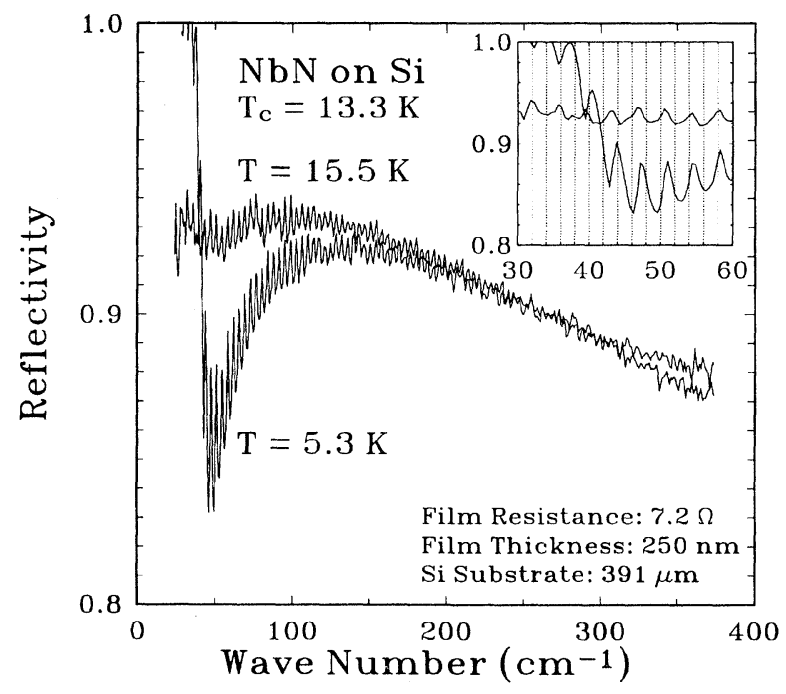

FIG. 3. The reflectivity of the $\mathrm{NbN}$ film on silicon above and below the superconducting transition temperature. The absolute accuracy of the reflectivity is $\pm 1 \%$ and of the temperature $\pm 0.5 \mathrm{~K}$. 
room-temperature reflectivity up to $5000 \mathrm{~cm}^{-1}$. The low reflectivity below $600 \mathrm{~cm}^{-1}$ for the $\mathrm{NbN}$ on a quartz substrate is a result of the poor film quality of this sample, which also showed no optical gap down to $5.3 \mathrm{~K}$. Figure 3 shows the reflectivity of the Si-substrate sample above and below $T_{c}$ with $0.5-\mathrm{cm}^{-1}$ resolution below $100 \mathrm{~cm}^{-1}$ and $1-\mathrm{cm}^{-1}$ resolution above. The interference fringes are seen to be very sharp. In particular, one can see in the inset that the phase of the fringes at $5.3 \mathrm{~K}$ change with respect to those at $15.5 \mathrm{~K}$ near $46 \mathrm{~cm}^{-1}$. Above 46 $\mathrm{cm}^{-1}$ the fringes are in phase, while below they are $180 .^{\circ}$ out of phase. This will be seen to be the frequency where the real and imaginary conductivities are equal at $5.3 \mathrm{~K}$. The reflectivity in the normal state is relatively low (due to the particular film thickness), making the superconductivity behavior very evident. Below the gap, the reflectivity at $5.3 \mathrm{~K}$ is $1.0 \pm 0.01$.

\section{iv. Calculations}

\section{A. Optical properties of a metal film on silicon}

Optical properties of the metal-film-on-silicon system have been analyzed using a standard procedure based on impedance mismatch. ${ }^{9}$ For a material with a refractive index $n$ and absorption coefficient $\alpha$, the impedance $\eta$ and the propagation coefficient $\gamma$ are defined as

$$
\begin{aligned}
& \eta=\left(\frac{\mu_{0}}{\epsilon_{0} \hat{\epsilon}}\right]^{1 / 2}=\frac{377 \Omega}{n+i \alpha / 4 \pi \bar{v}}, \\
& \gamma=i \omega\left(\mu_{o} \epsilon_{0} \hat{\epsilon}\right)^{1 / 2}=\frac{\alpha}{2}+2 \pi i n \bar{v},
\end{aligned}
$$

where $\bar{v}$ is the wave number $\left(\mathrm{cm}^{-1}\right)$. For a single layer of material described by $\eta_{2}$ and $\gamma_{2}$ and with thickness $d$, the load impedance seen at the first interface is

$$
Z_{L 1}=\eta_{2} \frac{\eta_{3} \cosh \left(\gamma_{2} d\right)+\eta_{2} \sinh \left(\gamma_{2} d\right)}{\eta_{2} \cosh \left(\gamma_{2} d\right)+\eta_{3} \sinh \left(\gamma_{2} d\right)},
$$

where $\eta_{3}$ is the impedance of the backing region. Then the reflectivity, transmission, and absorption of the system when light is incident from a medium with impedance $\eta_{1}$, are calculated using the formulas

$$
\begin{aligned}
& \hat{r}=\frac{Z_{L 1}-\eta_{1}}{Z_{L 1}+\eta_{1}}, \\
& R=\hat{r}^{*}, \\
& \hat{t}=\frac{2 Z_{L 1}}{Z_{L 1}+\eta_{1}}, \\
& T=\hat{t}^{*}, \\
& A=1-R-T .
\end{aligned}
$$

In a case where the absorption coefficient is negligible, the impedance $\eta$ is directly proportional to $1 / n$ and the equations for $R$ and $T$ reduce to the expected formulas.

In the case where the backing region is another dielectric layer, the value of $\eta_{3}$ in Eq. (3) is replaced by an effective load impedance, which is calculated using the same equations. This procedure can be repeated for any series of layers with different optical properties and thickness.

For a thin conducting film, the load impedance equation of Eq. (3) can be simplified by taking a limit as $d \rightarrow 0$. For such a film in air (impedance $\eta_{0}=377 \Omega$ ), the load impedance of the metal film is

$$
\begin{aligned}
Z_{L} & \approx \frac{\eta_{0}}{1+\eta_{0} d i \omega \hat{\epsilon} \epsilon_{0}}, \\
& \approx \frac{\eta_{0}}{1+\eta_{0} \sigma_{n} d}=\frac{\eta_{0}}{1+\eta_{0} / R_{\square}},
\end{aligned}
$$

where

$$
\hat{\epsilon}=\epsilon_{1}-i \epsilon_{2}=\epsilon_{1}-i \sigma_{n} / \omega \epsilon_{0},
$$

and $\sigma_{n}$ is the real part of the normal conductivity. $R_{\square}$ is the resistance, which would be measured across a square section of the film. For our system, consisting of conducting film on a silicon substrate, $\eta_{0}$ is replaced by the effective impedance of the silicon substrate $Z_{L, S}$ :

$$
Z_{L} \approx \frac{Z_{L, S}}{1+Z_{L, S} / R_{\square}},
$$

where

$$
Z_{L, S} \approx \eta_{s} \frac{\eta_{0} \cosh \left(\gamma_{s} d\right)+\eta_{s} \sinh \left(\gamma_{s} d\right)}{\eta_{s} \cosh \left(\gamma_{s} d\right)+\eta_{0} \sinh \left(\gamma_{s} d\right)},
$$

where $\eta_{s}$ is the silicon optical impedance and $d$ the silicon substrate thickness. Sample reflectivity, transmission, and absorption can now be calculated using Eqs. (4)-(8) above. Values of $n$ and $k$ for silicon were taken from Palik. ${ }^{10}$

Results of these calculations for the absolute reflectivity, transmission, and absorption as a function of the film resistance, $R_{\square}$, are shown in Fig. 4. Small values of $R_{\square}$ correspond to the relatively thick metallic layers,

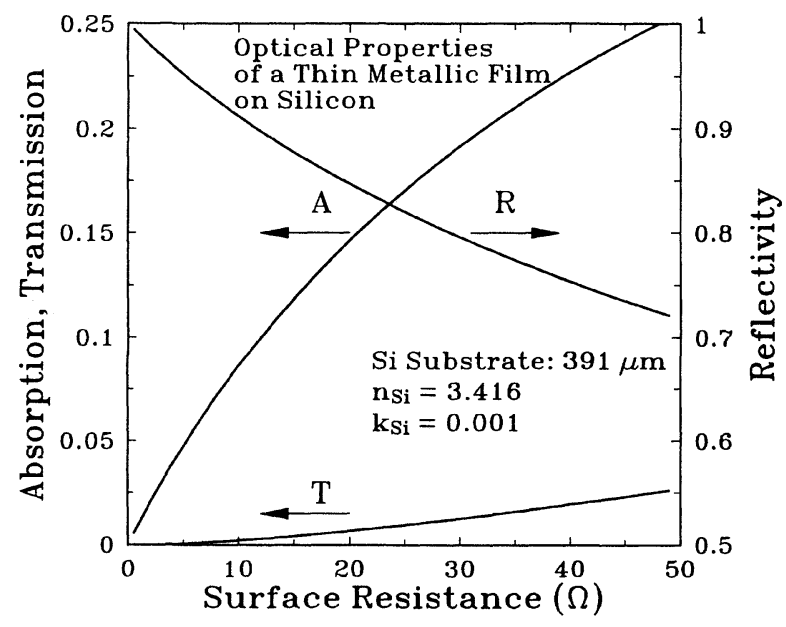

FIG. 4. The calculated absolute level of absorption $A$, reflectivity $R$, and transmission $T$ of a thin metal film on a silicon substrate vs surface resistance per square, $R_{\square}$. 
resulting in very high reflectivity and low transmission. As the film becomes thinner and the film resistance increases, there is some transmission by the film. Since we have measured an absolute value for the reflectivity of $R \approx 0.93$ at low frequencies in the normal state (see Fig. 3 ), it is possible to estimate the value of $R_{\square} \approx 7.2 \Omega$. As confirmation of this, Fig. 5 shows the calculated reflectivity versus wave number for the normal film on silicon, where both the interference fringe amplitude as well as the absolute reflectivity depend on $R_{\square}$. The agreement with experiment is good.

\section{B. Mattis-Bardeen theory}

The calculations of Mattis and Bardeen, ${ }^{2}$ for the extreme anomalous and dirty limit, have been used to find the ratio of the complex superconducting conductivity $\sigma_{s}$ to the normal conductivity $\sigma_{n}$, which is assumed constant:

$$
\frac{\sigma_{s}}{\sigma_{n}}=\frac{\sigma_{1}}{\sigma_{n}}-i \frac{\sigma_{2}}{\sigma_{n}} .
$$

This ratio applied to $R_{\square}$ in Eq. (12) gives the complex surface load impedance of the superconducting film on silicon:

$$
Z_{L} \approx \frac{Z_{L, S}}{1+\left(Z_{L, S} / R_{\square}\right)\left[\left(\sigma_{1}-i \sigma_{2}\right) / \sigma_{n}\right]} .
$$

The only input parameters in these calculations are the values of the energy gap $2 \Delta$, the temperature $T$, and the frequency $\omega$ (or wave number). Using the best-fit gap energy of $34.5 \mathrm{~cm}^{-1}$ at $5.3 \mathrm{~K}\left[2 \Delta(T=0 \mathrm{~K})=3.9 k_{B} T_{c}\right]$ gives the theoretical ratios for $\sigma_{1} / \sigma_{n}$ and $\sigma_{2} / \sigma_{n}$ shown in Fig. 6, for $T=5.3 \mathrm{~K}$, our lowest temperature. Figure

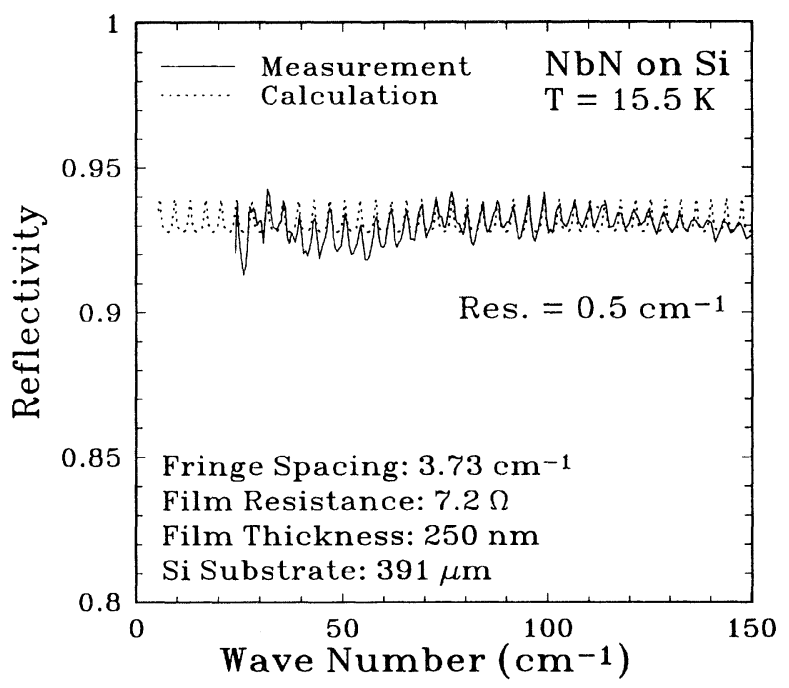

FIG. 5. The measured reflectivity of the $\mathrm{NbN}$ film on silicon at $15.5 \mathrm{~K}$ together with the calculated reflectivity $R$ of a thin metal film with surface resistance $R_{\square}$ of $7.2 \Omega / \square$ on a silicon substrate. Both the value of $R$ and the interference fringe amplitude depend on $R_{\square}$. (The film thickness is negligible and does not enter the equations.)

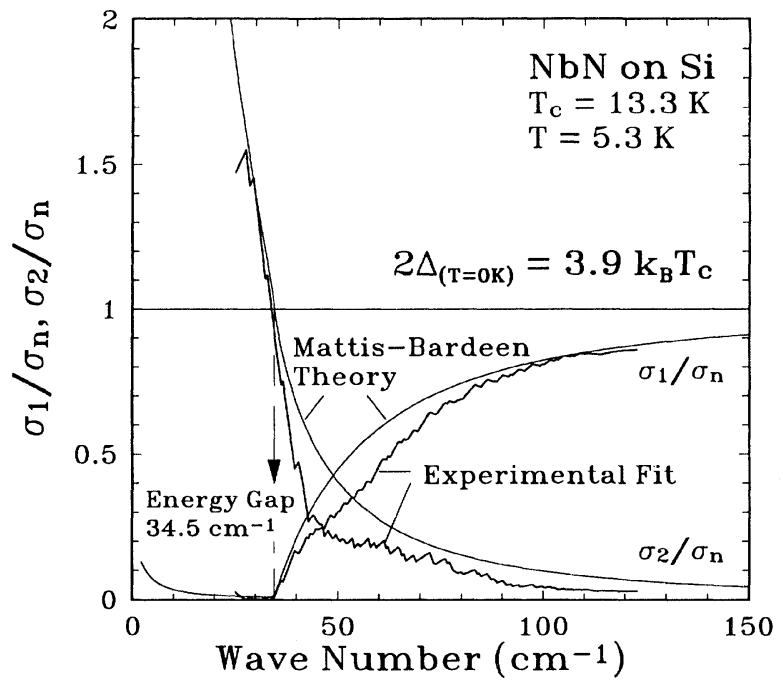

FIG. 6. The ratios of the complex superconducting conductivity at $5.3 \mathrm{~K}$ to the normal (real) conductivity, calculated using the Mattis-Bardeen theory (smooth lines), and obtained from a fit to the 5.3-K reflectivity data of Fig. 3.

7 shows the reflectivity at $5.3 \mathrm{~K}$ calculated (dotted line) with the Mattis-Bardeen (MB) conductivity curves of Fig. 6 compared with the measurement. Clearly there are similarities between the two spectra, including the phase shift in the interference fringes when $\sigma_{1}$ equals $\sigma_{2}$, so that the experimental and theoretical fringes are in phase throughout the spectrum. There is, however, a discrepancy in the overall magnitude of the reflectivity dip. If one starts with the MB values of $\sigma_{1}$ and $\sigma_{2}$ at high wave numbers, where they approach 1 and 0 , re-

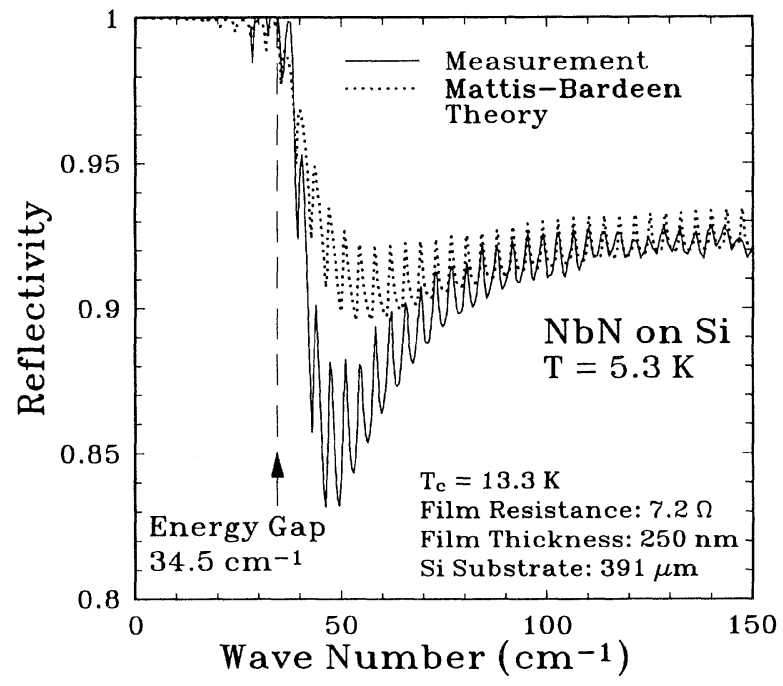

FIG. 7. The measured reflectivity of the $\mathrm{NbN}$ film on silicon, below $150 \mathrm{~cm}^{-1}$ at $5.3 \mathrm{~K}$, compared with that calculated using the Mattis-Bardeen complex conductivity of Fig. 6. The resolution is $0.5 \mathrm{~cm}^{-1}$. 


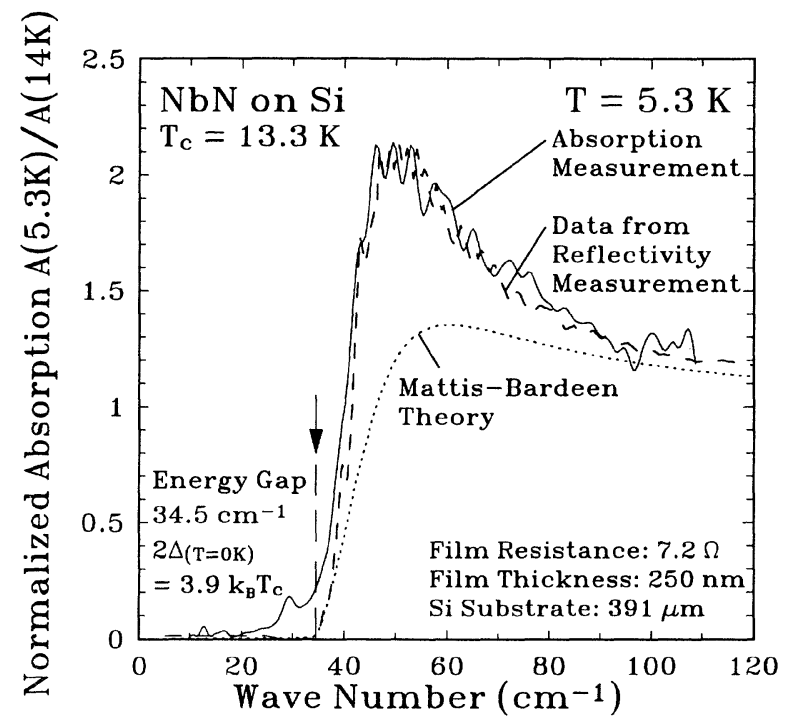

FIG. 8. The measured normalized absorption spectrum of the $\mathrm{NbN}$ film on silicon at $5.3 \mathrm{~K}$ (solid line), compared with that calculated (dotted line) using the Mattis-Bardeen complex conductivity of Fig. 6, and that calculated (dashed line) using the complex conductivity shown in Fig. 6, which was obtained by fitting to the measured reflectivity of Fig. 3.

spectively, and slowly reduces the wave number, one can find values of $\sigma_{1}$ and $\sigma_{2}$ that do not deviate too far from the MB values but give the best fit to the reflectivity data, including the correct phase and amplitude of the interference fringes, and these conductivities are shown in Fig. 6. (This best fit is virtually identical to the measured spectrum of Fig. 7 and is not shown.) One can see that the intersection of $\sigma_{1}$ and $\sigma_{2}$ at $46 \mathrm{~cm}^{-1}$ from the experimental fit, determined from the phase of the interference fringes, is close to that given by the theory, but the theoretical values are too high. These fitted values were then used to calculate the absorption $A$, and this is shown in Fig. 8 along with the measured spectrum and that calculated using the MB values of Fig. 6 with the interference removed. The agreement between the experimental $A$ curve and that obtained from the fitted $R$ curve is excellent and the MB theory shows a discrepancy similar to that seen in Fig. 7 .

\section{DISCUSSION}

The values of the complex conductivity calculated using the Mattis-Bardeen theory are too high for this $\mathrm{NbN}$ material. This finding agrees with the transmission re- sults of Ref. 3. The solution, however, does not appear to be the introduction of a scattering time $\tau$ into the normal conductivity, i.e.,

$$
\sigma_{n}=\frac{\sigma_{n 0}}{1+i \omega \tau}=\frac{\sigma_{n 0}}{1+i \bar{v} / \gamma},
$$

where $\bar{v}$ is in wave numbers.

Figure 3 shows the normal reflectivity decreasing with wave number, presumable due to such scattering events, but a fit at $350 \mathrm{~cm}^{-1}$ gives $\gamma$ equal to $400 \mathrm{~cm}^{-1}$, which would have a negligible effect at $40 \mathrm{~cm}^{-1}$ near the gap. In contrast, Karecki, Pena, and Perkowitz ${ }^{3}$ found it necessary to have $\gamma$ equal to $113 \mathrm{~cm}^{-1}\left(\tau=4.7 \times 10^{-14}\right.$ $\mathrm{sec} / \mathrm{rad})$, in order to reconcile the theory and experiment.

We were not able to obtain the real and imaginary parts of the superconducting conductivity by the usual Kramers-Kronig analysis of our power reflectivity because the latter is determined by the silicon substrate. We have shown, however, that by fitting the reflectivity data when it contains interference fringes, the phases of which depend on $\sigma_{1}$ and $\sigma_{2}$, then it is possible to extract $\sigma_{1}$ and $\sigma_{2}$, as long as they do not deviate too far from the theory.

We have no phonon data with which to try to fit strong-coupling theories, but it may be seen that there appears to be little phonon sructure discernible above the interference fringes in our measurements in contrast to lead, ${ }^{11}$ for instance. Furthermore, there is no evidence in our reflectivity data of the two phonon peaks at 120 and $360 \mathrm{~cm}^{-1}$ seen in the tunneling measurements of Ref. 5 . This may imply that the coupling in this material is weak, but it should be noted that the infrared phonon features are always very small, and one has to work hard to see them. By contrast the tunneling technique couples more strongly to phonons and is much more senstive to small features in the electrical response. The value of the $T=0$ $\mathrm{K}$ energy gap of $3.9 k_{B} T_{c}$ is slightly lower than that seen by a few other authors, but this may be merely the result of the relatively low $T_{c}$, since it has been shown ${ }^{12}$ that the prefactor is proportional to $T_{c}$.

\section{ACKNOWLEDGMENTS}

This work was supported by Grant Nos. 5-85653 and 55-97003 from the Natural Sciences and Engineering Research Council (NSERC) of Canada. The financial assistance of the James Clerk Maxwell Instrument Development Fund and access to experimental facilities at the Alberta Microelectronic Centre is also gratefully acknowledged. One of us (M.D.) acknowledges support from the Deutsche Forschungsgemeinschaft (DFG).
*Present address: Department of Electrical Engineering, University of Alberta, Edmonton, Alberta, Canada T6G 2G7.

${ }^{1}$ D. E. Oates, A. C. Anderson, C. C. Chin, J. S. Derov, G. Dresselhaus, and M. S. Dresselhaus, Phys. Rev. B 43, 7655 (1991).
${ }^{2}$ D. C. Mattis and J. Bardeen, Phys. Rev. 111, 412 (1958).

${ }^{3}$ D. Karecki, R. E. Pena, and S. Perkowitz, Phys. Rev. B 25, 1565 (1982).

${ }^{4}$ J. Geerk, U. Schneider, W. Bangert, H. Rietschel, F. Gompf, M. Gurvitch, J. Remeika, and J. M. Rowell, Physica B 135, 
187 (1985).

${ }^{5}$ K. E. Kihlstrom, R. W. Simon, and S. A. Wolf, Physica B 135 , 198 (1985)

${ }^{6}$ K. E. Kornelsen, J. E. Eldridge, H. H. Wang, and J. M. Williams, Solid State Commun. 76, 1009 (1990).

${ }^{7}$ J. Vaneldik, J. Vac. Sci. Technol. A 8, 1288 (1990).

${ }^{8}$ J. E. Eldridge and C. C. Homes, Infrared Phys. 29, 143 (1989).
${ }^{9}$ S. Ramo and J. R. Whinnery, Fields and Waves in Modern Radio, 2nd ed. (Wiley, New York, 1953).

${ }^{10}$ Handbook of Optical Constants of Solids, edited by E. D. Palik (Academic, New York, 1985), Vol. I.

${ }^{11}$ B. Farnworth and T. Timusk, Phys. Rev. B 10, 2799 (1974).

${ }^{12}$ B. Mitrovic, H. G. Zarate, and J. P. Carbotte, Phys. Rev. B 29, 184 (1984). 